\title{
On the lattice point covering problem in dimension 2
}

\author{
Fei Xue* \\ Institut für Mathematik \\ Technische Universität Berlin \\ D-10623 Berlin, Germany \\ xue@math.tu-berlin.de
}

Submitted: Oct 24, 2018; Accepted: Mar 15, 2019; Published: Apr 19, 2019

(C) The author. Released under the CC BY-ND license (International 4.0).

\begin{abstract}
In this paper we study the lattice point covering property of some regular polygons in dimension 2.
\end{abstract}

Mathematics Subject Classifications: 52C05, 52C07, 11H06

\section{Introduction}

Let $\mathcal{K}^{n}$ be the set of all convex bodies, i.e., compact convex sets, in the $n$-dimensional Euclidean space $\mathbb{R}^{n}$ with non-empty interior. We denote by $\mathcal{K}_{(o)}^{n} \subset \mathcal{K}^{n}$ the set of all convex bodies, having the origin as an interior point, i.e., $\mathbf{0} \in \operatorname{int}(K)$, and by $\mathcal{K}_{(s)}^{n} \subset \mathcal{K}_{(o)}^{n}$ those bodies which are symmetric with respect to 0 , i.e., $K=-K$.

We say that a convex body $K \in \mathcal{K}^{n}$ has the lattice point covering property, if $K$ contains a lattice point of $\mathbb{Z}^{n}$ in any position, i.e., in any translation and rotation of $K$.

There are several beautiful results about the lattice point covering property.

Theorem 1 (Niven \& Zuckerman,[4]). A triangle with sides of lengths $a, b, c$, with $a \leqslant$ $b \leqslant c$, has the lattice point covering property if and only if $2 \Delta(c-1) \geqslant c^{2}$ where $\Delta$ is the area of the triangle.

Theorem 2 (Niven \& Zuckerman,[4]). Let $a$ and $b$ be the distances between the pairs of opposite sides, say with $a \leqslant b$, of a parallelogram $A B C D$ with an interior angle $\gamma \leqslant \pi / 2$. The parallelogram has the lattice point covering property if and only if $a \geqslant 1$ and one of the following conditions hold:

(i) $b \geqslant \sqrt{2}$;

(ii) $b \leqslant \sqrt{2}$ and $\alpha+\beta+\gamma \leqslant \pi / 2$, where $\alpha=\arccos (a / \sqrt{2})$ and $\beta=\arccos (b / \sqrt{2})$.

*Supported by a PhD scholarship of the Berlin Mathematical School. 
Theorem 3 (Henk \& Tsintsifas,[3]). Let $\mathcal{E} \subset \mathbb{R}^{n}$ be an ellipsoid with semi-axes $\alpha_{i}$, $1 \leqslant i \leqslant n$. The following statements are equivalent:

(i) $\mathcal{E}$ contains a lattice point of $\mathbb{Z}^{n}$ in any position,

(ii) $\sum_{i=1}^{n} \frac{1}{\alpha_{i}^{2}} \leqslant 4$,

(iii) $\mathcal{E}$ contains a cube of edge length 1 .

Let $K \in \mathcal{K}^{n}$. Denote by $Z(K)$ the lattice point covering radius of $K$, i.e., the smallest positive number $r$, such that $r K$ has the lattice point covering property. We are here concerned with the lattice point covering properties of regular polygons. Let us denote by

$$
H_{n}=\operatorname{conv}\left\{\left(\cos \left(\frac{2 k \pi}{n}\right), \sin \left(\frac{2 k \pi}{n}\right)\right): k=0,1, \cdots, n-1\right\}
$$

the regular $n$-gon.

Our main result is:

Theorem 4. Let $t_{i}>0, i \in \mathbb{N}$.

(1) The following statements are equivalent:

(i) $t_{4 n} \cdot H_{4 n}$ contains a lattice point of $\mathbb{Z}^{2}$ in any position,

(ii) $t_{4 n} \cdot H_{4 n}$ contains a ball with radius $\frac{\sqrt{2}}{2}$,

(iii) $t_{4 n} \geqslant \frac{\frac{\sqrt{2}}{2}}{\cos \frac{\pi}{2 n}}$.

(2) The following statements are equivalent for $n=1,2$ :

(i) $t_{4 n+2} \cdot H_{4 n+2}$ contains a lattice point of $\mathbb{Z}^{2}$ in any position,

(ii) $t_{4 n+2} \cdot H_{4 n+2}$ contains $\left[-\frac{1}{2}, \frac{1}{2}\right]^{2}$,

(iii) $t_{6} \geqslant \frac{1}{3-\sqrt{3}} \approx 0.788675 \ldots ; t_{10} \geqslant \frac{\cos \frac{\pi}{5}-\sin \frac{\pi}{5}+\sin \frac{2 \pi}{5}-\cos \frac{2 \pi}{5}}{2 \sin \frac{\pi}{5}} \approx 0.734342 \ldots$

\section{Corollary 5.}

(i) $Z\left(H_{4 n}\right)=\frac{\frac{\sqrt{2}}{2}}{\cos \frac{\pi}{2 n}}$, for $n \in \mathbb{N}$.

(ii) $Z\left(H_{6}\right)=\frac{1}{3-\sqrt{3}}$.

(iii) $Z\left(H_{10}\right)=\frac{\cos \frac{\pi}{5}-\sin \frac{\pi}{5}+\sin \frac{2 \pi}{5}-\cos \frac{2 \pi}{5}}{2 \sin \frac{\pi}{5}}$.

This paper is organized as follows: in the Section 2 we will introduce the covering radius and the gauge function; then we will give a necessary and a sufficient condition of the lattice point covering property in the Section 3 and the Section 4, respectively; the content of the Section 5 will be the proof of the main theorem. 


\section{Covering Radius}

The covering radius of $K \in \mathcal{K}_{(o)}^{n}$ with respect to $\mathbb{Z}^{n}$ is denoted by

$$
c(K)=c\left(K, \mathbb{Z}^{n}\right)=\min \left\{\lambda>0: \lambda K+\mathbb{Z}^{n}=\mathbb{R}^{n}\right\} .
$$

The gauge function $\|\cdot\|$ associated to a $K \in \mathcal{K}_{(o)}^{n}$ is the function

$$
\|\cdot\|_{K}: \mathbb{R}^{n} \rightarrow[0, \infty)
$$

defined by $\|\boldsymbol{v}\|_{K}=\min \{t>0: \boldsymbol{v} \in t K\}$.

Theorem 6. Let $K \in \mathcal{K}_{(o)}^{n}$. Then $K$ contains a lattice point of $\mathbb{Z}^{n}$ in any position if and only if, for any $o(K)$ rotation of $K, c(o(K)) \leqslant 1$.

Proof. If $c(o(K))>1$ for some rotation $o(K)$, then there exists a point $\boldsymbol{x} \in \mathbb{R}^{n}$, such that for every $\boldsymbol{u} \in \mathbb{Z}^{2}$,

$$
\|\boldsymbol{x}-\boldsymbol{u}\|_{o(K)}>1 \Longleftrightarrow\|\boldsymbol{u}-\boldsymbol{x}\|_{-o(K)}>1 .
$$

Therefore, $-o(K)+\boldsymbol{x}$ does not contain a lattice point of $\mathbb{Z}^{2}$.

If $c(o(K)) \leqslant 1$ for any rotation $o(K)$, then for any point $\boldsymbol{x} \in \mathbb{R}^{n}$, since $o(K)+\mathbb{Z}^{n}=\mathbb{R}^{n}$, there exists a lattice point $\boldsymbol{u} \in \mathbb{Z}^{2}$, such that

$$
\|\boldsymbol{x}-\boldsymbol{u}\|_{o(K)} \leqslant 1 \Longleftrightarrow\|\boldsymbol{u}-\boldsymbol{x}\|_{-o(K)} \leqslant 1 .
$$

So, $-o(K)+\boldsymbol{x}$ contain a lattice point $u$.

Therefore, the lattice point covering property of a convex body depends on the covering radius of all rotations of this convex body.

\section{Necessary Condition}

According to the knowledge of the lattice covering for a centrally symmetric convex body, we have:

Theorem 7 (I.Fáry,[1]). Let $K \in \mathcal{K}_{(s)}^{2}$, such that $K+\mathbb{Z}^{2}$ is a lattice covering. Then $K$ contains a spacefiller $L$, i.e., a parallelogram or a centrally symmetric hexagon, such that $L+\mathbb{Z}^{2}$ is a lattice tiling.

Since the lattice point covering property depends on the lattice covering of all rotations, we have:

Theorem 8. Let $K \in \mathcal{K}_{(s)}^{2}$. Then $K$ contains a lattice point of $\mathbb{Z}^{2}$ in any position, if and only if

(1) $o(K)+\mathbb{Z}^{2}=\mathbb{R}^{2}$

(2) o(K) contains a spacefiller $L$, i.e., parallelogram or a centrally symmetric hexagon, such that $L+\mathbb{Z}^{2}$ is a lattice tiling,

for any rotation $o(K)$. 
Proof. Apply Theorem 6 and 7.

We here call it a necessary condition of the lattice point covering property, because this condition has to hold, but is uneasy to check.

\section{Sufficient Condition}

For a planar convex body, it is possible to check some inscribed parallelograms, i.e., by checking the Steiner symmetrization of the convex body. We will give a sufficient condition of the lattice point covering property in this way.

The Steiner symmetrization of $K \in \mathcal{K}^{2}$ with respect to $\left\{\boldsymbol{x} \in \mathbb{R}^{2}: x_{2}=0\right\}$, denoted by $\operatorname{St}_{1}(K)$, is a convex body symmetric with respect to $\left\{\boldsymbol{x} \in \mathbb{R}^{2}: x_{2}=0\right\}$, such that for each line $l$ vertical to $\left\{\boldsymbol{x} \in \mathbb{R}^{2}: x_{2}=0\right\}$,

$$
\operatorname{vol}_{1}(K \cap l)=\operatorname{vol}_{1}\left(\operatorname{St}_{1}(K) \cap l\right),
$$

where $\operatorname{vol}_{1}(L)$ denotes the length of a line segment $L$. For more information on the Steiner symmetrization, we refer to [2, Section 9.1]. It is obvious that $\operatorname{St}_{1}(K) \subset \operatorname{St}_{1}(L)$ for two convex bodies $K \subset L$.

Theorem 9. Let $K \in \mathcal{K}_{(s)}^{2}$. If for each rotation $o(K)$ of $K, \operatorname{St}_{1}(o(K))$ contains $\left[-\frac{1}{2}, \frac{1}{2}\right]^{2}$, then $K$ contains a lattice point of $\mathbb{Z}^{2}$ in any position.

Proof. Notice that if $\operatorname{St}_{1}(o(K))$ contains $\left[-\frac{1}{2}, \frac{1}{2}\right]^{2}$, then $o(K)$ contains a parallelogram in the form of $L=\operatorname{conv}\left\{\left(\frac{1}{2}, a\right),\left(\frac{1}{2}, a+1\right),\left(-\frac{1}{2},-a\right),\left(-\frac{1}{2},-a-1\right)\right\}$, which is a spacefiller with respect to $\mathbb{Z}^{2}$. Therefore $K$ has the lattice point covering property (cf. Theorem 6 ).

We also have the following proposition of lattice covering for sets symmetric with respect to the axis $o x$ and $o y$.

Proposition 10. Let $K \in \mathcal{K}_{(s)}^{2}$. If $K$ is symmetric with respect to $\left\{\boldsymbol{x} \in \mathbb{R}^{2}: x_{1}=0\right\}$ and $\left\{\boldsymbol{x} \in \mathbb{R}^{2}: x_{2}=0\right\}$, then $K+\mathbb{Z}^{2}=\mathbb{R}^{2}$ if and only if $K$ contains $\left[-\frac{1}{2}, \frac{1}{2}\right]^{2}$.

Proof. If $K$ contains $\left[-\frac{1}{2}, \frac{1}{2}\right]^{2}$, then $K+\mathbb{Z}^{2}$ is a lattice covering. Otherwise, if $K$ does not contain $\left[-\frac{1}{2}, \frac{1}{2}\right]^{2}$, i.e., $\left(\frac{1}{2}, \frac{1}{2}\right) \notin K$, then since $K$ is symmetric with respect to $\left\{\boldsymbol{x} \in \mathbb{R}^{2}\right.$ : $\left.x_{1}=0\right\}$ and $\left\{\boldsymbol{x} \in \mathbb{R}^{2}: x_{2}=0\right\}, K$ does not contain any point of $\left(\frac{1}{2}, \frac{1}{2}\right)+\mathbb{Z}^{2}$, thus $K+\mathbb{Z}^{2}$ does not contain $\left(\frac{1}{2}, \frac{1}{2}\right)+\mathbb{Z}^{2}$.

\section{Proof of the Main Theorem}

In this section we discuss the lattice point covering property of some regular polygons. The proofs have the following steps:

1. Prove that the Steiner symmetrizations of all rotations of the convex body contain $\left[-\frac{1}{2}, \frac{1}{2}\right]^{2}$ (using Theorem 9). 
2. Prove that a smaller copy of the convex body does not have the lattice point covering property (using Theorem 8(1) and Proposition 10).

We first look at the regular $4 n$-gon.

Proof of Theorem 4(1). If $t \cdot H_{4 n}$ contains $B_{2}\left(\frac{\sqrt{2}}{2}\right)$, where $B_{2}(r)$ denotes the Euclidean disk of radius $r$ centered at $\mathbf{0}$, then each rotation $o\left(t \cdot H_{4 n}\right)$ also contains $B_{2}\left(\frac{\sqrt{2}}{2}\right)$. Notice that $B_{2}\left(\frac{\sqrt{2}}{2}\right)$ contains $\left[-\frac{1}{2}, \frac{1}{2}\right]^{2}$, therefore $o\left(t \cdot H_{4 n}\right)+\mathbb{Z}^{2}$ is always a lattice covering, thus $t \cdot H_{4 n}$ has the lattice point covering property (cf. Theorem 6 ).

If $t \cdot H_{4 n}$ does not contain $B_{2}\left(\frac{\sqrt{2}}{2}\right)$, then $o\left(t \cdot H_{4 n}^{\prime}, \frac{\pi}{4}\right)+\left(\frac{1}{2}, \frac{1}{2}\right)$, does not contain any lattice point of $\mathbb{Z}^{2}$, where $o\left(t \cdot H_{4 n}^{\prime}, \frac{\pi}{4}\right)$ is the rotation of $t \cdot H_{4 n}^{\prime}$ by angle $\frac{\pi}{4}$.

Denote by $o(K, \theta)$ the counterclockwise rotation of $K$ by angle $\theta$, i.e.,

$$
o\left(H_{n}, \theta\right)=\operatorname{conv}\left\{\left(\cos \left(\frac{2 k \pi}{n}+\theta\right), \sin \left(\frac{2 k \pi}{n}+\theta\right)\right): k=0,1, \cdots, n-1\right\} .
$$

Then we look at the regular hexagon.

Proof of Theorem 4(2), $n=1$. For the symmetric reason between $H_{6}$ and $\mathbb{Z}^{2}$, the case $\frac{\pi}{12} \leqslant \theta \leqslant \frac{\pi}{6}$ is actually symmetric to the case $0 \leqslant \theta \leqslant \frac{\pi}{12}$ with respect to the line $\left\{\boldsymbol{x} \in \mathbb{R}^{2}: x_{2}=x_{1}\right\}$. We are going to prove that $\operatorname{St}_{1}\left(o\left(\frac{1}{3-\sqrt{3}} H_{6}, \theta\right)\right)$ contains $\left[-\frac{1}{2}, \frac{1}{2}\right]^{2}$ for $0 \leqslant \theta \leqslant \frac{\pi}{12}$ (cf. Theorem 9).

By calculation,

$$
\begin{aligned}
\operatorname{St}_{1}\left(o\left(H_{6}, \theta\right)\right)=\operatorname{conv} & \left\{( \pm \cos \theta, 0),\left( \pm \cos \left(\theta-\frac{\pi}{3}\right), \pm \frac{\sin \frac{\pi}{3}}{2 \sin \left(\theta+\frac{\pi}{6}\right)}\right),\right. \\
& \left.\left( \pm \cos \left(\theta+\frac{\pi}{3}\right), \pm \frac{\sqrt{3}}{2 \cos \theta}\right)\right\},
\end{aligned}
$$

which is also symmetric with respect to $\left\{\boldsymbol{x} \in \mathbb{R}^{2}: x_{1}=0\right\}$. In order to check whether $\operatorname{St}_{1}\left(o\left(\frac{1}{3-\sqrt{3}} H_{6}, \theta\right)\right)$ contains $\left[-\frac{1}{2}, \frac{1}{2}\right]^{2}$ for $0 \leqslant \theta \leqslant \frac{\pi}{12}$, notice that when $0 \leqslant \theta \leqslant \frac{\pi}{12}$, it holds

$$
\cos \left(\theta+\frac{\pi}{3}\right) \leqslant \frac{\sqrt{3}}{2 \cos \theta}
$$

(cf. Proposition 12), therefore the line $\left\{\boldsymbol{x} \in \mathbb{R}^{2}: x_{2}=x_{1}\right\}$ may intersect the boundary of $\mathrm{St}_{1}\left(o\left(H_{6}, \theta\right)\right)$ with the edge

$$
\operatorname{conv}\left\{(\cos \theta, 0),\left(\cos \left(\theta-\frac{\pi}{3}\right), \frac{\sin \frac{\pi}{3}}{2 \sin \left(\theta+\frac{\pi}{6}\right)}\right)\right\}
$$

or the edge

$$
\operatorname{conv}\left\{\left(\cos \left(\theta-\frac{\pi}{3}\right), \frac{\sin \frac{\pi}{3}}{2 \sin \left(\theta+\frac{\pi}{6}\right)}\right),\left(\cos \left(\theta+\frac{\pi}{3}\right), \frac{\sqrt{3}}{2 \cos \theta}\right)\right\} .
$$


Case 1: $\cos \left(\theta-\frac{\pi}{3}\right) \leqslant \frac{\sin \frac{\pi}{3}}{2 \sin \left(\theta+\frac{\pi}{6}\right)}$, i.e., $0 \leqslant \theta \leqslant \arcsin \left(\frac{\sqrt[4]{3}}{2}\right)-\frac{\pi}{6}$.

In this case, the line $\left\{\boldsymbol{x} \in \mathbb{R}^{2}: x_{2}=x_{1}\right\}$ intersects the edge

$$
\operatorname{conv}\left\{(\cos \theta, 0),\left(\cos \left(\theta-\frac{\pi}{3}\right), \frac{\sin \frac{\pi}{3}}{2 \sin \left(\theta+\frac{\pi}{6}\right)}\right)\right\}
$$

with $(s(\theta), s(\theta))$, where

$$
\frac{s(\theta)}{s(\theta)-\cos \theta}=\frac{\frac{\sin \frac{\pi}{3}}{2 \sin \left(\theta+\frac{\pi}{6}\right)}}{\cos \left(\theta-\frac{\pi}{3}\right)-\cos \theta}
$$

thus

$$
s(\theta)=\frac{\sin \frac{\pi}{3} \cos \theta}{\sin \frac{\pi}{3}-2 \sin ^{2}\left(\theta+\frac{\pi}{6}\right)+2 \cos \theta \sin \left(\theta+\frac{\pi}{6}\right)} .
$$

This function $s(\theta)$ is increasing in $\left[0, \arcsin \left(\frac{\sqrt[4]{3}}{2}\right)-\frac{\pi}{6}\right]$ (cf. Proposition 13), therefore,

and

$$
s(\theta) \geqslant s(0)=\frac{\sqrt{3}}{\sqrt{3}+1},
$$

$$
\frac{1}{3-\sqrt{3}} s(\theta) \geqslant \frac{1}{2}
$$

So $\operatorname{St}_{1}\left(o\left(\frac{1}{3-\sqrt{3}} H_{6}, \theta\right)\right)$ always contains $\left[-\frac{1}{2}, \frac{1}{2}\right]^{2}$ when $\theta \in\left[0, \arcsin \left(\frac{\sqrt[4]{3}}{2}\right)-\frac{\pi}{6}\right]$.

Case 2: $\cos \left(\theta-\frac{\pi}{3}\right) \geqslant \frac{\sin \frac{\pi}{3}}{2 \sin \left(\theta+\frac{\pi}{6}\right)}$, i.e., $\arcsin \left(\frac{\sqrt[4]{3}}{2}\right)-\frac{\pi}{6} \leqslant \theta \leqslant \frac{\pi}{12}$.

In this case, the line $\left\{\boldsymbol{x} \in \mathbb{R}^{2}: x_{2}=x_{1}\right\}$ intersects the edge

$$
\operatorname{conv}\left\{\left(\cos \left(\theta-\frac{\pi}{3}\right), \frac{\sin \frac{\pi}{3}}{2 \sin \left(\theta+\frac{\pi}{6}\right)}\right),\left(\cos \left(\theta+\frac{\pi}{3}\right), \frac{\sqrt{3}}{2 \cos \theta}\right)\right\} \text {. }
$$

with $(t(\theta), t(\theta))$, where

$$
\frac{2 t(\theta)-\frac{\sqrt{3}}{\cos \theta}}{t(\theta)-\cos \left(\theta+\frac{\pi}{3}\right)}=\frac{\frac{\sin \frac{\pi}{3}}{\sin \left(\theta+\frac{\pi}{6}\right)}-\frac{\sqrt{3}}{\cos \theta}}{\cos \left(\theta-\frac{\pi}{3}\right)-\cos \left(\theta+\frac{\pi}{3}\right)},
$$

i.e.,

$$
t(\theta)=\frac{2 \sqrt{3} \sin \left(\theta+\frac{\pi}{6}\right)+\sqrt{3} \cos \left(\theta+\frac{\pi}{3}\right)}{4 \cos \theta \sin \left(\theta+\frac{\pi}{6}\right)+\sqrt{3}} .
$$

This function $t(\theta)$ is decreasing in $\left[\arcsin \left(\frac{\sqrt[4]{3}}{2}\right)-\frac{\pi}{6}, \frac{\pi}{12}\right]$, and in fact it is decreasing in $\left[\arcsin \left(\frac{\sqrt[4]{3}}{2}\right)-\frac{\pi}{6}, \frac{\pi}{6}\right]$ (cf. Proposition 14), therefore

$$
t(\theta) \geqslant t\left(\frac{\pi}{12}\right)>t\left(\frac{\pi}{6}\right)=\frac{\sqrt{3}}{\sqrt{3}+1},
$$


and

$$
\frac{1}{3-\sqrt{3}} t(\theta)>\frac{1}{2}
$$

Thus $\operatorname{St}_{1}\left(o\left(\frac{1}{3-\sqrt{3}} H_{6}, \theta\right)\right)$ always contains $\left[-\frac{1}{2}, \frac{1}{2}\right]^{2}$ when $\theta \in\left[\arcsin \left(\frac{\sqrt[4]{3}}{2}\right)-\frac{\pi}{6}, \frac{\pi}{12}\right]$.

To see that $\frac{1}{3-\sqrt{3}}$ is the minimum number, we refer to Proposition 10 applied to $\rho H_{6}$ for any $\rho<\frac{1}{3-\sqrt{3}}$.

Now we look at the regular 10-gon.

Proof of Theorem 4(2), $n=2$. For the symmetric reason between $H_{10}$ and $\mathbb{Z}^{2}$, the case $\frac{\pi}{20} \leqslant \theta \leqslant \frac{\pi}{10}$ is actually symmetric to the case $0 \leqslant \theta \leqslant \frac{\pi}{20}$ with respect to the line $\left\{\boldsymbol{x} \in \mathbb{R}^{2}: x_{2}=x_{1}\right\}$. We are going to prove that $\operatorname{St}_{1}\left(o\left(\frac{\cos \frac{\pi}{5}-\sin \frac{\pi}{5}+\sin \frac{2 \pi}{5}-\cos \frac{2 \pi}{5}}{2 \sin \frac{\pi}{5}} H_{10}, \theta\right)\right)$ contains $\left[-\frac{1}{2}, \frac{1}{2}\right]^{2}$ for $0 \leqslant \theta \leqslant \frac{\pi}{20}$ (cf. Theorem 9 ).

By calculation,

$$
\begin{aligned}
\operatorname{St}_{1}\left(o\left(H_{10}, \theta\right)\right)=\operatorname{conv}\{ & \pm(\cos \theta, 0), \\
& \left( \pm \cos \left(\theta-\frac{\pi}{5}\right), \pm \frac{\sin \frac{\pi}{10} \sin \frac{\pi}{5}}{\sin \left(\frac{\pi}{10}+\theta\right)}\right),\left( \pm \cos \left(\theta+\frac{\pi}{5}\right), \pm \frac{\sin \frac{\pi}{5} \sin \frac{3 \pi}{10}}{\sin \left(\frac{3 \pi}{10}-\theta\right)}\right) \\
& \left.\left( \pm \cos \left(\theta-\frac{2 \pi}{5}\right), \pm \frac{\sin \frac{3 \pi}{10} \sin \frac{2 \pi}{5}}{\sin \left(\frac{3 \pi}{10}+\theta\right)}\right),\left( \pm \cos \left(\theta+\frac{2 \pi}{5}\right), \pm \frac{\sin \frac{2 \pi}{5}}{\cos \theta}\right)\right\}
\end{aligned}
$$

While $\theta \in\left[0, \frac{\pi}{20}\right]$, it holds

$$
\begin{aligned}
\cos \left(\theta-\frac{\pi}{5}\right) & >\frac{\sin \frac{\pi}{10} \sin \frac{\pi}{5}}{\sin \left(\frac{\pi}{10}+\theta\right)} & \text { (cf. Proposition 16) } \\
\cos \left(\theta+\frac{\pi}{5}\right) & >\frac{\sin \frac{\pi}{5} \sin \frac{3 \pi}{10}}{\sin \left(\frac{3 \pi}{10}-\theta\right)} & \text { (cf. Proposition 17) } \\
\cos \left(\theta-\frac{2 \pi}{5}\right) & <\frac{\sin \frac{3 \pi}{10} \sin \frac{2 \pi}{5}}{\sin \left(\frac{3 \pi}{10}+\theta\right)} & \text { (cf. Proposition 18) } \\
\cos \left(\theta+\frac{2 \pi}{5}\right) & <\frac{\sin \frac{2 \pi}{5}}{\cos \theta} & \text { (cf. Proposition 19) } .
\end{aligned}
$$

Therefore, the line $\left\{\boldsymbol{x} \in \mathbb{R}^{2}: x_{2}=x_{1}\right\}$ intersects $\operatorname{St}_{1}\left(o\left(H_{10}, \theta\right)\right)$ with the edge

$$
\operatorname{conv}\left\{\left(\cos \left(\theta+\frac{\pi}{5}\right), \frac{\sin \frac{\pi}{5} \sin \frac{3 \pi}{10}}{\sin \left(\frac{3 \pi}{10}-\theta\right)}\right),\left(\cos \left(\theta-\frac{2 \pi}{5}\right), \frac{\sin \frac{3 \pi}{10} \sin \frac{2 \pi}{5}}{\sin \left(\frac{3 \pi}{10}+\theta\right)}\right)\right\}
$$

at the point $(t(\theta), t(\theta))$, where

$$
\frac{t(\theta)-\frac{\sin \frac{3 \pi}{10} \sin \frac{2 \pi}{5}}{\sin \left(\frac{3 \pi}{10}+\theta\right)}}{t(\theta)-\cos \left(\theta-\frac{2 \pi}{5}\right)}=\frac{\frac{\sin \frac{\pi}{5} \sin \frac{3 \pi}{10}}{\sin \left(\frac{3 \pi}{10}-\theta\right)}-\frac{\sin \frac{3 \pi}{10} \sin \frac{2 \pi}{5}}{\sin \left(\frac{3 \pi}{10}+\theta\right)}}{\cos \left(\theta+\frac{\pi}{5}\right)-\cos \left(\theta-\frac{2 \pi}{5}\right)} .
$$


The function $t(\theta)$ is increasing in $\left[0, \frac{\pi}{20}\right]$ (cf. Proposition 15), therefore

$$
t(\theta) \geqslant t(0)=\frac{\sin \frac{\pi}{5}}{\cos \frac{\pi}{5}-\sin \frac{\pi}{5}+\sin \frac{2 \pi}{5}-\cos \frac{2 \pi}{5}},
$$

and

$$
\frac{\cos \frac{\pi}{5}-\sin \frac{\pi}{5}+\sin \frac{2 \pi}{5}-\cos \frac{2 \pi}{5}}{2 \sin \frac{\pi}{5}} t(\theta) \geqslant \frac{1}{2} .
$$

So $\operatorname{St}_{1}\left(o\left(\frac{\cos \frac{\pi}{5}-\sin \frac{\pi}{5}+\sin \frac{2 \pi}{5}-\cos \frac{2 \pi}{5}}{2 \sin \frac{\pi}{5}} H_{10}, \theta\right)\right)$ always contains $\left[-\frac{1}{2}, \frac{1}{2}\right]^{2}$ when $\theta \in\left[0, \frac{\pi}{20}\right]$.

To see that $\frac{\cos \frac{\pi}{5}-\sin \frac{\pi}{5}+\sin \frac{2 \pi}{5}-\cos \frac{2 \pi}{5}}{2 \sin \frac{\pi}{5}}$ is the minimum number, we refer to Proposition 10 applied to $\rho H_{10}$ for any $\rho<\frac{\cos \frac{\pi}{5}-\sin \frac{\pi}{5}+\sin \frac{2 \pi}{5}-\cos \frac{2 \pi}{5}}{2 \sin \frac{\pi}{5}}$.

Remark 11. $\mathrm{St}_{1}\left(o\left(H_{2 n}, \theta\right)\right), \theta \in\left[0, \frac{\pi}{4 n}\right]$, has the vertices

$$
\begin{aligned}
& (\cos \theta, 0), \\
& \left(\cos \left(\theta-\frac{2 \pi}{n}\right), \frac{\sin \frac{\pi}{n} \sin \frac{2 \pi}{n}}{\sin \left(\frac{\pi}{n}+\theta\right)}\right),\left(\cos \left(\theta+\frac{2 \pi}{n}\right), \frac{\sin \frac{2 \pi}{n} \sin \frac{3 \pi}{n}}{\sin \left(\frac{3 \pi}{n}-\theta\right)},\right. \\
& \left(\cos \left(\theta-\frac{4 \pi}{n}\right), \frac{\sin \frac{3 \pi}{n} \sin \frac{4 \pi}{n}}{\sin \left(\frac{3 \pi}{n}+\theta\right)}\right),\left(\cos \left(\theta+\frac{4 \pi}{n}\right), \frac{\sin \frac{4 \pi}{n} \sin \frac{5 \pi}{n}}{\sin \left(\frac{5 \pi}{n}-\theta\right)}\right) \ldots
\end{aligned}
$$

One can deal with all regular $(4 n+2)$-gons similarly.

\section{A Some Inequalities}

\section{Proposition 12.}

for $0 \leqslant \theta \leqslant \frac{\pi}{12}$.

$$
\cos \left(\theta+\frac{\pi}{3}\right) \leqslant \frac{\sqrt{3}}{2 \cos \theta}
$$

Proof. It is equivalent to

$$
\begin{gathered}
2 \cos \theta \cos \left(\theta+\frac{\pi}{3}\right) \leqslant \sqrt{3} \\
\Longleftrightarrow \cos \frac{\pi}{3}+\cos \left(2 \theta+\frac{\pi}{3}\right) \leqslant \sqrt{3} \\
\Longleftrightarrow \cos \left(2 \theta+\frac{\pi}{3}\right) \leqslant \sqrt{3}-\frac{1}{2},
\end{gathered}
$$

which holds true since $\cos \left(2 \theta+\frac{\pi}{3}\right)$ is decreasing in $\theta \in\left[0, \frac{\pi}{12}\right]$ and $\cos \frac{\pi}{3}<\sqrt{3}-\frac{1}{2}$.

\section{Proposition 13.}

$$
s(\theta)=\frac{\sin \frac{\pi}{3} \cos \theta}{\sin \frac{\pi}{3}-2 \sin ^{2}\left(\theta+\frac{\pi}{6}\right)+2 \cos \theta \sin \left(\theta+\frac{\pi}{6}\right)} \geqslant s(0)
$$

for $0 \leqslant \theta \leqslant \arcsin \left(\frac{\sqrt[4]{3}}{2}\right)-\frac{\pi}{6}$ 
Proof. Notice that

$$
s(\theta)=\frac{\sqrt{3} \cos \theta}{-3+\sqrt{3}+4 \cos ^{2} \theta} .
$$

Since $\frac{x}{-3+\sqrt{3}+4 x^{2}}$ is decreasing in $x \in\left[\cos \left(\arcsin \left(\frac{\sqrt[4]{3}}{2}\right)-\frac{\pi}{6}\right), 1\right]$ and $\cos \theta$ is decreasing in $\theta \in\left[0, \arcsin \left(\frac{\sqrt[4]{3}}{2}\right)-\frac{\pi}{6}\right]$ then $s(\theta)$ is increasing.

\section{Proposition 14.}

$$
t(\theta)=\frac{2 \sqrt{3} \sin \left(\theta+\frac{\pi}{6}\right)+\sqrt{3} \cos \left(\theta+\frac{\pi}{3}\right)}{4 \cos \theta \sin \left(\theta+\frac{\pi}{6}\right)+\sqrt{3}} \geqslant t\left(\frac{\pi}{6}\right)
$$

for $\arcsin \left(\frac{\sqrt[4]{3}}{2}\right)-\frac{\pi}{6} \leqslant \theta \leqslant \frac{\pi}{6}$.

Proof. Notice that

$$
t(\theta)=\frac{3 \sin \left(\theta+\frac{\pi}{3}\right)}{4 \sin ^{2}\left(\theta+\frac{\pi}{3}\right)+\sqrt{3}-1} .
$$

Since $\frac{x}{4 x^{2}+\sqrt{3}-1}$ is decreasing in $x \in\left[\sin \left(\arcsin \left(\frac{\sqrt[4]{3}}{2}\right)-\frac{\pi}{6}\right), \frac{1}{2}\right]$ and $\sin \left(\theta+\frac{\pi}{3}\right)$ is increasing in $\theta \in\left[\arcsin \left(\frac{\sqrt[4]{3}}{2}\right)-\frac{\pi}{6}, \frac{\pi}{6}\right]$ then $t(\theta)$ is decreasing.

\section{Proposition 15.}

$$
t(\theta) \geqslant t(0)
$$

for $0 \leqslant \theta \leqslant \frac{\pi}{20}$ where

$$
\frac{t(\theta)-\frac{\sin \frac{3 \pi}{10} \sin \frac{2 \pi}{5}}{\sin \left(\frac{3 \pi}{10}+\theta\right)}}{t(\theta)-\cos \left(\theta-\frac{2 \pi}{5}\right)}=\frac{\frac{\sin \frac{\pi}{5} \sin \frac{3 \pi}{10}}{\sin \left(\frac{3 \pi}{10}-\theta\right)}-\frac{\sin \frac{3 \pi}{10} \sin \frac{2 \pi}{5}}{\sin \left(\frac{3 \pi}{10}+\theta\right)}}{\cos \left(\theta+\frac{\pi}{5}\right)-\cos \left(\theta-\frac{2 \pi}{5}\right)}
$$

Proof. Notice that

$$
t(\theta)=\frac{2 \sin \frac{3 \pi}{10} \cos \frac{\pi}{10} \cos \theta}{2 \cos ^{2} \theta+\sin \frac{3 \pi}{5}-\cos \frac{3 \pi}{5}-1} .
$$

Since $\frac{x}{2 x^{2}+\sin \frac{3 \pi}{5}-\cos \frac{3 \pi}{5}-1}$ is decreasing in $x \in\left[\cos \frac{\pi}{20}, 1\right]$ and $\cos \theta$ is decreasing in $\theta \in\left[0, \frac{\pi}{20}\right]$ thus $t(\theta)$ is increasing.

\section{Proposition 16.}

$$
\cos \left(\theta-\frac{\pi}{5}\right)>\frac{\sin \frac{\pi}{10} \sin \frac{\pi}{5}}{\sin \left(\frac{\pi}{10}+\theta\right)}
$$

for $0 \leqslant \theta \leqslant \frac{\pi}{20}$. 
Proof. The statement is equivalent to

$$
\begin{aligned}
& \sin \left(\frac{\pi}{10}+\theta\right) \cos \left(\theta-\frac{\pi}{5}\right)>\sin \frac{\pi}{10} \sin \frac{\pi}{5} \\
\Leftrightarrow & \sin \left(2 \theta-\frac{\pi}{10}\right)+\sin \frac{3 \pi}{10}>2 \sin \frac{\pi}{10} \sin \frac{\pi}{5} \\
\Leftrightarrow & \sin \left(2 \theta-\frac{\pi}{10}\right)>2 \sin \frac{\pi}{10} \sin \frac{\pi}{5}-\sin \frac{3 \pi}{10} .
\end{aligned}
$$

Since $\sin \left(2 \theta-\frac{\pi}{10}\right)$ is increasing for $0 \leqslant \theta \leqslant \frac{\pi}{20}$, and

$$
\sin \frac{3 \pi}{10}-\sin \frac{\pi}{10}-2 \sin \frac{\pi}{10} \sin \frac{\pi}{5}>0,
$$

the inequality holds for $0 \leqslant \theta \leqslant \frac{\pi}{20}$.

Proposition 17.

$$
\cos \left(\theta+\frac{\pi}{5}\right)>\frac{\sin \frac{\pi}{5} \sin \frac{3 \pi}{10}}{\sin \left(\frac{3 \pi}{10}-\theta\right)}
$$

for $0 \leqslant \theta \leqslant \frac{\pi}{20}$.

Proof. The statement is equivalent to

$$
\begin{aligned}
& \sin \left(\frac{3 \pi}{10}-\theta\right) \cos \left(\theta+\frac{\pi}{5}\right)>\sin \frac{\pi}{5} \sin \frac{3 \pi}{10} \\
\Leftrightarrow & \sin \frac{\pi}{2}+\sin \left(\frac{\pi}{10}-2 \theta\right)>2 \sin \frac{\pi}{5} \sin \frac{3 \pi}{10} \\
\Leftrightarrow & \sin \left(\frac{\pi}{10}-2 \theta\right)>2 \sin \frac{\pi}{5} \sin \frac{3 \pi}{10}-\sin \frac{\pi}{2} .
\end{aligned}
$$

Since $\sin \left(\frac{\pi}{10}-2 \theta\right)$ is decreasing for $0 \leqslant \theta \leqslant \frac{\pi}{20}$, and

$$
0>2 \sin \frac{\pi}{5} \sin \frac{3 \pi}{10}-\sin \frac{\pi}{2},
$$

the inequality holds for $0 \leqslant \theta \leqslant \frac{\pi}{20}$.

\section{Proposition 18.}

$$
\cos \left(\theta-\frac{2 \pi}{5}\right)<\frac{\sin \frac{3 \pi}{10} \sin \frac{2 \pi}{5}}{\sin \left(\frac{3 \pi}{10}+\theta\right)}
$$

for $0 \leqslant \theta \leqslant \frac{\pi}{20}$.

Proof. The statement is equivalent to

$$
\begin{aligned}
& \sin \left(\frac{3 \pi}{10}+\theta\right) \cos \left(\theta-\frac{2 \pi}{5}\right)<\sin \frac{3 \pi}{10} \sin \frac{2 \pi}{5} \\
\Leftrightarrow & \sin \left(2 \theta-\frac{\pi}{10}\right)+\sin \frac{7 \pi}{10}<2 \sin \frac{3 \pi}{10} \sin \frac{2 \pi}{5} \\
\Leftrightarrow & \sin \left(2 \theta-\frac{\pi}{10}\right)<2 \sin \frac{3 \pi}{10} \sin \frac{2 \pi}{5}-\sin \frac{7 \pi}{10}
\end{aligned}
$$


Since $\sin \left(2 \theta-\frac{\pi}{10}\right)$ is increasing for $0 \leqslant \theta \leqslant \frac{\pi}{20}$, and

$$
0<2 \sin \frac{3 \pi}{10} \sin \frac{2 \pi}{5}-\sin \frac{7 \pi}{10}
$$

the inequality holds for $0 \leqslant \theta \leqslant \frac{\pi}{20}$.

Proposition 19.

$$
\cos \left(\theta+\frac{2 \pi}{5}\right)<\frac{\sin \frac{2 \pi}{5}}{\cos \theta}
$$

for $0 \leqslant \theta \leqslant \frac{\pi}{20}$.

Proof. The statement is equivalent to

$$
\begin{aligned}
& \cos \theta \cos \left(\theta+\frac{2 \pi}{5}\right)<\sin \frac{2 \pi}{5} \\
\Leftrightarrow & \cos \left(2 \theta+\frac{2 \pi}{5}\right)+\cos \frac{2 \pi}{5}<2 \sin \frac{2 \pi}{5} \\
\Leftrightarrow & \cos \left(2 \theta+\frac{2 \pi}{5}\right)<2 \sin \frac{2 \pi}{5}-\cos \frac{2 \pi}{5} .
\end{aligned}
$$

Since $\cos \left(2 \theta+\frac{2 \pi}{5}\right)$ is decreasing for $0 \leqslant \theta \leqslant \frac{\pi}{20}$, and

$$
\cos \frac{2 \pi}{5}<2 \sin \frac{2 \pi}{5}-\cos \frac{2 \pi}{5},
$$

the inequality holds for $0 \leqslant \theta \leqslant \frac{\pi}{20}$.

\section{References}

[1] I.FÁRY, Sur la densité des réseaux de domaines convexes, Bull. Soc. Math. France, 178, (1950), 152-161.

[2] P.M.Gruber, Convex and Discrete Geometry. Springer, Berlin Heidelberg, 2007.

[3] M.Henk, G.A.Tsintsifas, Lattice Point Coverings, Chinese Advances in Mathematics, 36(4), (2007), 441-446.

[4] I.Niven, H.S.Zuckerman, Lattice Point Coverings by Plane Figures, Amer. Math. Monthly, 74(4), (1967), 353-362. 